\title{
A comparison of sedimentary aliphatic hydrocarbon distribution between East China Sea and southern Okinawa Trough
}

\author{
Woei-Lih Jeng ${ }^{\mathrm{a}, *}$, Chih-An Huh ${ }^{\mathrm{b}}$ \\ ${ }^{a}$ Institute of Oceanography, National Taiwan University, Taipei, Taiwan, ROC \\ ${ }^{\mathrm{b}}$ Institute of Earth Sciences, Academia Sinica, Taipei, Taiwan, ROC
}

Received 5 July 2007; received in revised form 15 October 2007; accepted 19 November 2007

Available online 4 December 2007

\begin{abstract}
In this study, 34 surface sediments were collected from the East China Sea (ECS) shelf, and their aliphatic hydrocarbon concentrations measured. The hydrocarbon distribution of the ECS was compared with that of the southern Okinawa Trough (SOT) to explore for possible relations between the two coastal marine areas. Results from principal component analysis performed on hydrocarbon data of ECS and SOT sediments indicated that the first two components explained $74 \%$ of the total variance and that the score plot of the two components showed three groups. The first group defined by the 12 SOT sediments contained three sediments on the ECS inner shelf. Eight sediments except one on the ECS inner shelf formed group II, and the remaining 21 sediments located away from the inner shelf (mainly on the mid-to-outer shelf) formed group III. Even though three inner shelf sediments cluster with group I, the separation between groups I and II suggests that the hydrocarbon relation between the inner shelf and the SOT is very weak. Groups II and III do not cluster together; their separation is supported by previous studies showing that Changjiang River sediment tends to remain in the coastal waters and is transported to the south by the coastal currents along the Chinese coast. Also, group I does not cluster with group III, signifying a great difference between them; this can be attributed to little material exchange between the ECS and the SOT because of the Kuroshio Current. In addition, the SOT and ECS inner shelf had carbon preference index values averaging $2.92 \pm 0.79(n=12)$ and $4.56 \pm 0.93$ $(n=8)$, terrestrial/aquatic ratios averaging $9.64 \pm 4.33(n=12)$ and $50.4 \pm 58.0(n=8)$, and $\left(n \mathrm{C}_{27}+n \mathrm{C}_{29}+n \mathrm{C}_{31}\right) / \mathrm{TOC}$ ratios averaging $1731 \pm 482 \times 10^{-7} \mathrm{~g}(\mathrm{gC})^{-1}(n=12)$ and $3016 \pm 868 \times 10^{-7} \mathrm{~g}(\mathrm{gC})^{-1}(n=8)$, respectively; strongly indicating considerable differences between the two areas. Based on the present results, hydrocarbon export from the ECS inner shelf to the SOT appears to be unlikely. (C) 2007 Elsevier Ltd. All rights reserved.
\end{abstract}

Keywords: Sediment; Hydrocarbon; East China Sea; Southern Okinawa Trough

\section{Introduction}

The main circulation patterns of the Changjiang Estuary and adjacent East China Sea (ECS) can be characterized by the northward flow of warm $\left(13^{\circ} \mathrm{C}\right)$ and saline $(34 \mathrm{psu})$ waters of the Taiwan Warm Current (TWC) and a southward flow of the colder $\left(5^{\circ} \mathrm{C}\right)$ and less saline (30 psu) waters of the Yellow Sea Coastal Current (YSCC) (Fig. 1). During high river runoff, one part of the Changjiang plume with the freshest water extends to the south along the coast, and the other part with low salinity

\footnotetext{
${ }^{*}$ Corresponding author. Tel.: + 886223636040x301; fax: +886223626092 .

E-mail address: wljeng@oc.ntu.edu.tw (W.-L. Jeng).
}

extends offshore on average toward the northeast. However, during low river runoff, the surface plume spreads toward the south only, possibly because of the influence of the TWC and YSCC convergence (Beardsley et al., 1985).

The ECS has a very broad continental shelf. The Changjiang River annually discharges $\sim 5 \times 10^{8}$ tons of sediment directly into the ECS (Milliman and Meade, 1983). This sediment is confined to the coastal zones of the ECS and ultimately transported south and southwestward by the Changjiang Coastal Water (Milliman et al., 1985); offshore transport has been prevented by tidal currents and by the northward movement of the Taiwan Warm Water (Milliman et al., 1989). DeMaster et al. (1985) have reported that the accumulation rates of ECS shelf sediments are relatively high in muddy areas and low in 


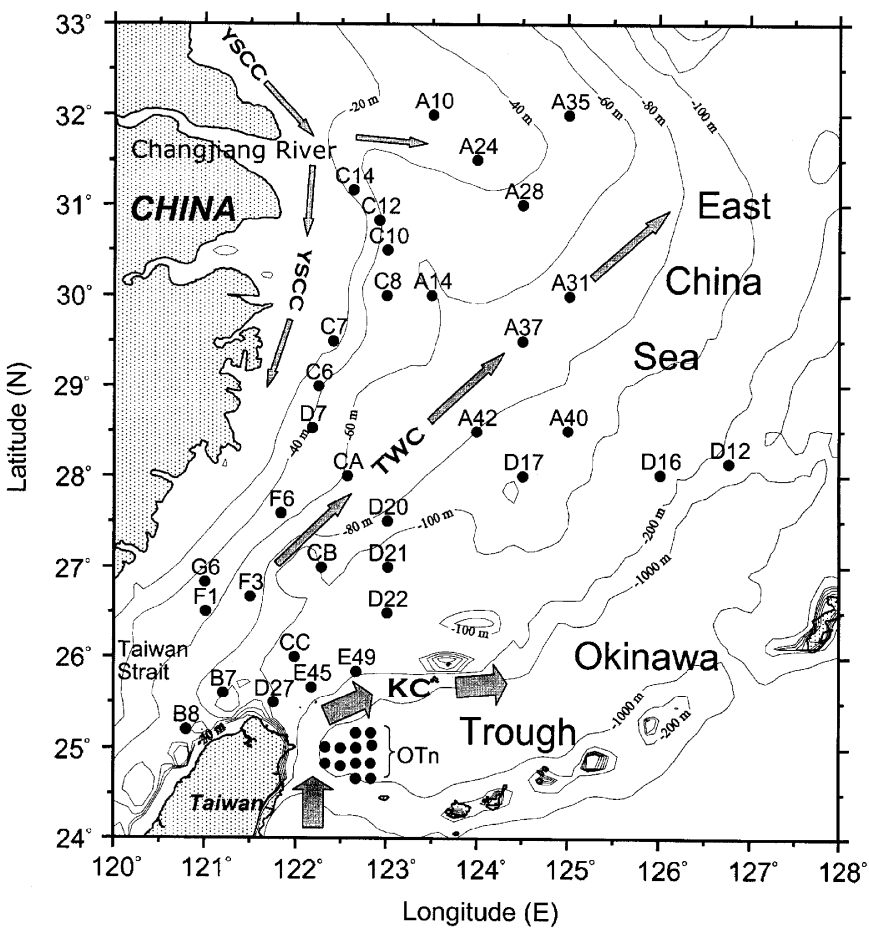

Fig. 1. Sample sites on the East China Sea shelf and in the Okinawa Trough with water circulation patterns. Three major currents are the Yellow Sea Coastal Current (YSCC), the Taiwan Warm Current (TWC) and the Kuroshio Current (KC). Sample codes for the Okinawa Trough (OTn) are top row (from left): stations 18 and 5; second row: stations 1, 3, 4 and 22; third row: stations 12, 23, 19 and 9; bottom row: stations 20 and 8 .

sandy areas. The sedimentation rates on the ECS shelf range from $\sim 2$ to $0.02 \mathrm{~cm} \mathrm{yr}^{-1}$, and generally decrease southward along the inner shelf and eastward offshore (Huh and $\mathrm{Su}, 1999$ ). On the other hand, the southern Okinawa Trough (SOT) has high sedimentation rates located approximately in the lower trough, deeper than $1000 \mathrm{~m}$ (range from 0.25 to $0.52 \mathrm{~cm} \mathrm{yr}^{-1}$ estimated from the excess ${ }^{210} \mathrm{~Pb}$ profiles of cores), while low sedimentation rates are generally in the upper trough, shallower than $1000 \mathrm{~m}$ (Chung and Chang, 1995). Based on results from a site $\left(24^{\circ} 48.24^{\prime} \mathrm{N}, 122^{\circ} 30.00^{\prime} \mathrm{E}\right)$ of ODP Leg 195 in the SOT, the sedimentation rate has always been high, reaching $325 \mathrm{~cm} \mathrm{kyr}^{-1}\left(0.325 \mathrm{~cm} \mathrm{y}^{-1}\right)$ since the late Holocene (ODP, 2001). Therefore, the SOT is apparently an area of focused sedimentation along the path of the Kuroshio Current (KC). Furthermore, the lower slope $(>1000 \mathrm{~m})$ sediments consist almost entirely of silty mud (Chen et al., 1995); for instance, mud accounts for over 97\% (Jeng and Chen, 1995), while the upper slope $(<1000 \mathrm{~m})$ sediments are composed of sand with little mud (Chen et al., 1995).

Transport processes of particulate matter in the marginal seas were first investigated in the middle Atlantic Bight during the SEEP (Shelf Edge Exchange Processes I and II) program (Walsh et al., 1988; Biscaye et al., 1988) and later in several other seas in the world. For the ECS and the SOT, it is the KEEP (Kuroshio Edge Exchange Processes) program (Wong et al., 2000; Liu et al., 2003). Using the results of field observations and diagnostic numerical experiments, Yanagi et al. (1996) have concluded that suspended matter is transported from the shelf edge to the inner shelf in summer and from the inner shelf to the shelf edge in autumn (which is maximum) and winter due to the vertical circulation mainly induced by the monsoon wind. Studying time-series sediment trap samples collected at SST-1 $\left(29^{\circ} 21.6^{\prime} \mathrm{N}, 128^{\circ} 13.5^{\prime} \mathrm{E}, 1100 \mathrm{~m}\right.$ water depth), Katayama and Watanabe (2003) have concluded that lateral transport through the bottom layer is important in the transport of terrigenous particles from the ECS shelf to the mid-OT and that Changjiang influence is relatively high during winter. As far as organic carbon is concerned, analyzing 11 sediment cores from the mid-ECS, Oguri et al. (2003) have reported that organic carbon accumulation rates range from 0.42 to $3.1 \mathrm{mg} \mathrm{C} \mathrm{cm}^{-2} \mathrm{yr}^{-1}$ and are higher in the inner shelf edge and the slope areas. Based on the balance of organic carbon budget in the shelf sediments, Kao et al. (2003) consider that a major fraction of adsorbed carbon from primary production must be exported out of the ECS shelf. They further propose that one possible mechanism is the cross-shelf export of particulate organic matter (POM) and that the most likely site for deposition of exported POM from the ECS is the SOT. Liu et al. (2000) propose a conceptual model illustrating the possible route of transporting sediment. From isotopic evidence, Kao et al. (2003) conclude that a major fraction of the sedimentary organic matter in the SOT may originate from the inner shelf of the ECS.

In the present study, 34 surface sediments from the ECS were analyzed for their aliphatic hydrocarbons. A comparison of hydrocarbon distribution in sediments between ECS and SOT (data from Jeng and Huh, 2006) was made in order to understand the significance of cross-shelf export of particulate organic carbon to the SOT. To find the possible relation of hydrocarbon distribution between the two areas, principal component analysis (PCA) was employed since it was a data reduction technique used to simplify data sets while retaining the underlying patterns within the data. This result of comparing hydrocarbon similarity between ECS and SOT may shed some light on the transport of sedimentary lipids between them, and might provide some information about the question "Does the ECS export organic carbon to the SOT?"

\section{Experimental}

In the present study, 34 surface sediments were collected from the ECS shelf on board R/V Ocean Researcher I using a box corer (Fig. 1). The top 3-4 cm of sediment was stored in stainless steel boxes (pre-cleaned with solvent) and kept frozen $\left(-20^{\circ} \mathrm{C}\right)$ until analyzed. Prefixes A, B, C, D, E, F and $\mathrm{G}$ of sample codes denote cruise numbers 551 (May 29-June 4, 1999), 542 (March 8-11, 1999), 525 (August 17-24, 1998), 493 (July 8-13, 1997), 460 (August 20-26, 1996), 456 (July 6-12, 1996) and 417 (April 24-May 1, 1995), respectively. 
In the lab, frozen sediments were freeze-dried. Following the addition of an internal standard $\left(n-\mathrm{C}_{24} \mathrm{D}_{50}\right)$, the dried sediment was extracted with a mixture of dichloromethane and methanol $(1: 1 ; \mathrm{v} / \mathrm{v})$ for $24 \mathrm{~h}$ in a Soxhlet apparatus. The lipid extract was then saponified by reflux for $3 \mathrm{~h}$ with $0.5 \mathrm{~N} \mathrm{KOH}$ solution in methanol. The non-saponifiable lipids were isolated by hexane extraction four times and concentrated using $\mathrm{N}_{2}$ gas. The aliphatic hydrocarbon fraction was isolated from the neutral lipids by silica gel (deactivated with $5 \% \mathrm{H}_{2} \mathrm{O}$ ) column chromatography using hexanes.

For gas chromatography (GC) analysis, an HP 5890A gas chromatograph equipped with a split/splitless injector and a flame ionization detector (FID) was used. Separation of aliphatic hydrocarbons was achieved by an SPB-1 capillary column $(30 \mathrm{~m} \times 0.25 \mathrm{~mm}$ i.d. $\times 0.25 \mu \mathrm{m})$. Oven temperature programming was $45-90^{\circ} \mathrm{C}$ at $15^{\circ} \mathrm{Cmin}^{-1}$ and $90-270^{\circ} \mathrm{C}$ at $3{ }^{\circ} \mathrm{Cmin}^{-1}$ for analyzing aliphatic hydrocarbons. Identification was made with co-injection of authentic standards and gas chromatography-mass spectrometry (GC-MS). The GC-MS analyses were performed with an HP 6890 GC (HP-1MS crosslinked methyl siloxane column, $30 \mathrm{~m} \times 0.25 \mathrm{~mm}$ i.d. $\times 0.25 \mu \mathrm{m}$ ) interfaced directly to an HP 5973 quadrupole mass selective detector (electron ionization, electron energy $70 \mathrm{eV}$, scanned from 50 to $550 \mathrm{Da}$ ).

An SGE (Australia) OCI-5 cool on-column injector was also fitted in the gas chromatograph for obtaining the best quantification. GC peak areas of all hydrocarbons and the internal standard were obtained using an electronic integrator (Chromatopac C-R6A, Shimadzu, Japan). Each hydrocarbon concentration was determined using the internal standard. Based on eight replicate analyses, the analytical precision (expressed as the percent coefficient of variation) of hydrocarbon abundances was calculated to be $2-8 \%$.

All sediment samples were determined for their total organic carbon by the dichromate-acid oxidation method (Gaudette et al., 1974) modified by addition of $\mathrm{Ag}_{2} \mathrm{SO}_{4}$ to $\mathrm{H}_{2} \mathrm{SO}_{4}$ at the rate of $15 \mathrm{~g} \mathrm{~L}^{-1}$. Titration was carried out with an automatic titrator (Metrohm 702 SM Titrino, Switzerland). The relative standard deviation of TOC determination was generally $<1 \%$.

PCA was performed on GC data of aliphatic hydrocarbons. Each compound was used as a variable. Prior to PCA, all hydrocarbon data were standardized by subtracting each data from the mean and dividing by the standard deviation. PCA was performed using xISTAT version 5.1 (Addinsoft, France).

\section{Results and discussion}

From the GC chromatograms of aliphatic hydrocarbons, the sediments showed a general picture of similar distribution patterns with $n$-alkanes predominant along with squalene, diploptene, UCM (unresolved complex mixtures), etc. (Fig. 2). In general, higher-molecular-weight $n$-alkanes of terrigenous sources $-\mathrm{C}_{25}, \mathrm{C}_{27}, \mathrm{C}_{29}$ and $\mathrm{C}_{31}$ (Rieley et al., 1991) dominated over lower-molecularweight ones of marine sources such as phytoplankton$\mathrm{C}_{15}, \mathrm{C}_{17}, \mathrm{C}_{19}$ and $\mathrm{C}_{21}$ (Winters et al., 1969; Blumer et al., 1971). This result can be attributed to $n$-alkanes derived from marine sources being degraded faster than those from terrigenous ones (Meyers et al., 1984; Gagosian and Peltzer, 1986). Also, land plants have proportionally larger amounts of hydrocarbons than do marine algae. Squalene exhibited a wide concentration range as compared to $n$ alkanes (Table 1) probably because it is a lipid constituent of most marine organisms and particularly of phytoplankton (Bieger et al., 1997; Salas et al., 2006). Also, it possesses relatively high stability like $n-\mathrm{C}_{18}$ and pristane (Jeng and Huh, 2004). Diploptene (hop-22(29)-ene) is derived from terrestrial higher plants and is also formed by bacteria (Rohmer et al., 1984). If higher plants are the sole source of diploptene, a strong correlation between diploptene and terrestrial higher plant $n$-alkanes has been demonstrated (Prahl et al., 1992). A positive correlation between diploptene and higher plant $n$-alkanes was found for 34 ECS sediments $\left(\sum \mathrm{C}_{25-33}=-152+13.2 \mathrm{Di}, r=0.96\right)$, strongly indicating a higher plant source to the sediments. UCM, consisting of cyclic and branched alkanes, is known to resist microbial degradation more effectively than $n$-alkanes and thus has a greater tendency to remain in the environment after $n$-alkanes have degraded (Gough and Rowland, 1990; Bouloubassi and Saliot, 1993). It has a linkage to degraded or weathered petroleum residues (Venkatesan et al., 1980; Readman et al., 1987). Most marine sediments analyzed generally contained a medium UCM (Fig. 2; Table 1). This does not necessarily reflect the presence of degraded petroleum in the sediments since in some cases concentrations of $\mathrm{UCM}<10 \mu \mathrm{gg}^{-1}$ are common in coastal marine environments far from petrogenic hydrocarbon sources (Matsumoto, 1983; Tolosa et al., 1996). In addition, the distribution of the lower molecular weight $n$-alkanes $\left(<\mathrm{C}_{23}\right)$ looked like a petrogenic origin (carbon preference index (CPI))close to 1) which could probably be attributed to recycled organic matter since the mid-to-outer shelf was composed mainly of relict sediments (Niino and Emery, 1961). Further, only five stations gave $\mathrm{Pr} / \mathrm{Ph}$ ratios close to 1 (A14, 0.82; $\mathrm{B} 7,0.81 ; \mathrm{D} 16,0.86 ; \mathrm{D} 27$, 1.21; E49, 1.05; calculated from Table 1). Combining the $\mathrm{UCM}$ and $\mathrm{Pr} / \mathrm{Ph}$ ratio results, it can be concluded that the petrogenic hydrocarbon contamination on the ECS shelf is minimal.

The sediment textures varied greatly as reflected by the TOC content of sediments ranging from 0.043 to $0.85 \mathrm{~g} \mathrm{C}(100 \mathrm{~g})^{-1}$ (Table 2). To compensate for the grain size difference, all hydrocarbon data were normalized to TOC before performing multivariate analysis.

Prior to PCA, cluster analysis was performed on all variables (e.g. individual aliphatic hydrocarbons) of ECS and SOT sediments (Tables 1 and 3). The resulting dendrogram (not shown) illustrated that UCM, diploptene, and squalene merged with the clusters in the final stages 

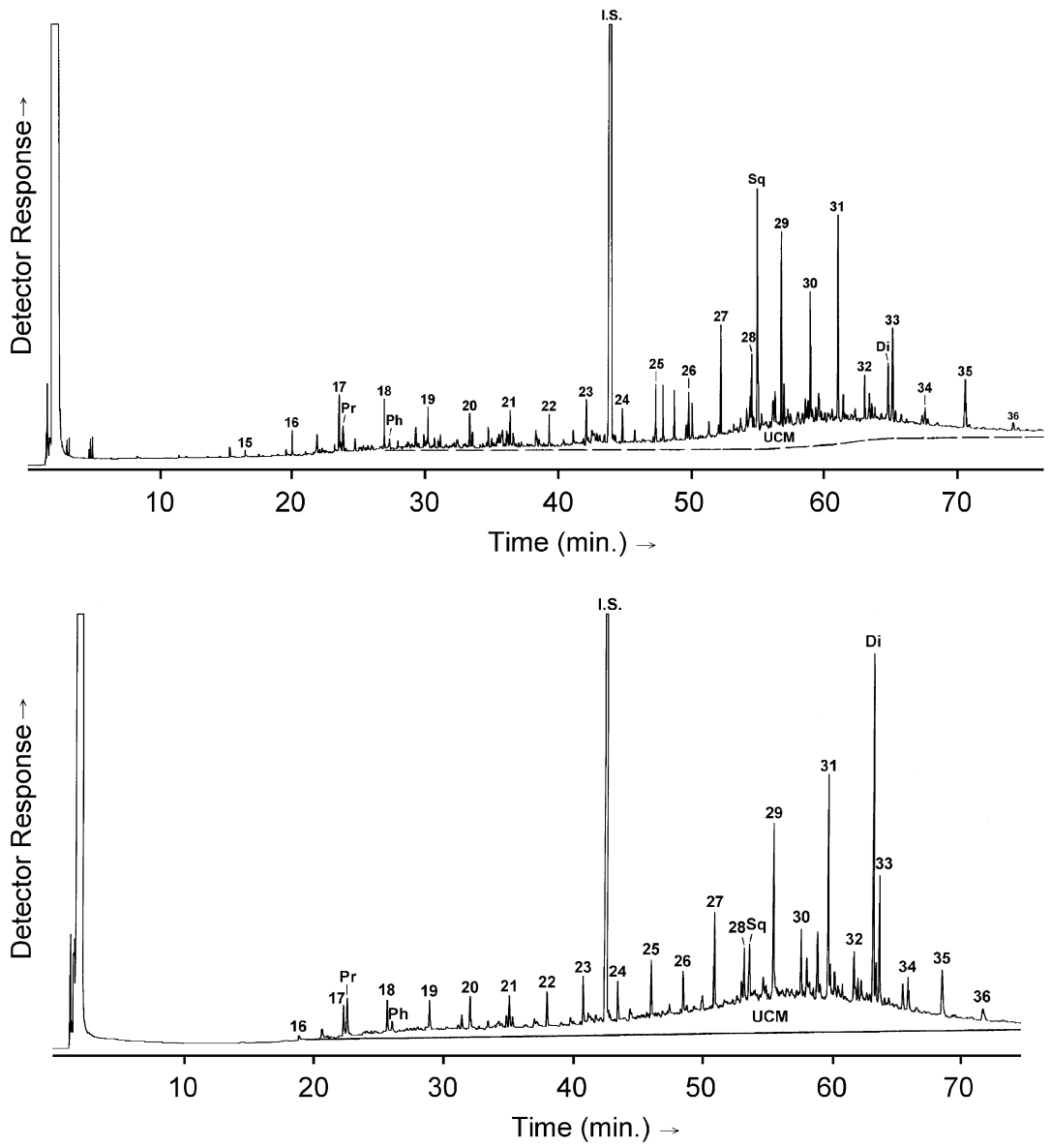

Fig. 2. Gas chromatograms of the aliphatic hydrocarbon fraction from station A40 (upper) and OT8 (lower). Numbers denote carbon numbers of $n$-alkanes. I.S., internal standard; Pr, pristane; Ph, phytane; Sq, squalene; Di, diploptene; and UCM, unresolved complex mixtures.

Table 1

Hydrocarbon concentrations $\left(\mathrm{ng} \mathrm{g}^{-1}\right.$ ) for East China Sea (ECS) sediments

\begin{tabular}{|c|c|c|c|c|c|c|c|c|c|}
\hline \multirow[t]{2}{*}{ Sample } & \multicolumn{9}{|c|}{ Aliphatic hydrocarbons ${ }^{\mathrm{a}}$} \\
\hline & $\mathrm{C}_{15}$ & $\mathrm{C}_{16}$ & $\mathrm{C}_{17}$ & $\operatorname{Pr}$ & $\mathrm{C}_{18}$ & $\mathrm{Ph}$ & $\mathrm{C}_{19}$ & $\mathrm{C}_{20}$ & $\mathrm{C}_{21}$ \\
\hline A10 & - & 1.66 & 4.27 & 5.95 & 3.99 & 1.42 & 2.96 & 2.37 & 7.78 \\
\hline A14 & - & - & 6.80 & 3.13 & 12.2 & 3.83 & 13.5 & 12.6 & 20.3 \\
\hline A24 & - & 5.19 & 32.1 & 41.8 & 15.1 & 5.81 & 16.6 & 7.91 & 11.8 \\
\hline A28 & 0.372 & 0.521 & 0.397 & - & 2.97 & 0.818 & 3.15 & 3.07 & 3.72 \\
\hline A31 & 2.17 & 7.09 & 15.7 & 13.1 & 13.1 & 4.99 & 12.4 & 10.3 & 16.0 \\
\hline A35 & - & - & 3.67 & 2.60 & 8.64 & - & 10.1 & 10.5 & 16.4 \\
\hline A 37 & 4.11 & 10.3 & 24.4 & 18.4 & 19.0 & 6.36 & 17.0 & 12.7 & 21.0 \\
\hline $\mathrm{A} 40$ & 0.789 & 4.90 & 12.7 & 10.3 & 11.7 & 2.33 & 9.34 & 8.97 & 10.1 \\
\hline A42 & 3.36 & 13.3 & 30.4 & 22.2 & 26.9 & 9.89 & 21.1 & 17.0 & 22.6 \\
\hline B7 & - & - & 2.10 & 1.68 & 5.39 & 2.07 & 5.26 & 5.60 & 7.42 \\
\hline B8 & - & 12.5 & 38.7 & 45.6 & 33.9 & 13.4 & 32.8 & 28.1 & 35.1 \\
\hline C6 & - & - & - & - & 11.2 & - & 25.8 & 24.8 & 41.7 \\
\hline C7 & 2.88 & 14.7 & 42.9 & 50.0 & 27.8 & 9.85 & 39.7 & 23.1 & 37.8 \\
\hline C8 & - & 4.34 & 17.2 & 11.8 & 17.5 & 6.36 & 16.8 & 13.7 & 19.7 \\
\hline $\mathrm{C} 10$ & - & - & 3.66 & 1.30 & 9.34 & - & 13.2 & 13.6 & 21.4 \\
\hline $\mathrm{C} 12$ & - & - & - & - & - & - & 10.3 & 18.4 & 34.4 \\
\hline C14 & - & - & - & - & - & - & 11.5 & 16.3 & 29.4 \\
\hline $\mathrm{CA}$ & - & - & 2.29 & - & 8.74 & 3.01 & 13.2 & 13.9 & 19.8 \\
\hline CB & - & - & 1.93 & 1.13 & 4.82 & 1.76 & 5.24 & 5.06 & 7.35 \\
\hline $\mathrm{CC}$ & - & - & - & - & 1.72 & - & 2.37 & 2.91 & 3.21 \\
\hline D7 & 25.4 & 32.1 & 70.8 & 62.6 & 42.5 & 16.7 & 50.6 & 32.7 & 57.3 \\
\hline D12 & - & 3.11 & 12.1 & 8.05 & 13.4 & 5.26 & 10.2 & 9.46 & 11.1 \\
\hline
\end{tabular}


Table 1 (continued)

\begin{tabular}{|c|c|c|c|c|c|c|c|c|c|}
\hline \multirow[t]{2}{*}{ Sample } & \multicolumn{9}{|c|}{ Aliphatic hydrocarbons $^{\mathrm{a}}$} \\
\hline & $\mathrm{C}_{15}$ & $\mathrm{C}_{16}$ & $\mathrm{C}_{17}$ & $\operatorname{Pr}$ & $\mathrm{C}_{18}$ & $\mathrm{Ph}$ & $\mathrm{C}_{19}$ & $\mathrm{C}_{20}$ & $\mathrm{C}_{21}$ \\
\hline D16 & 0 & 0.918 & 5.33 & 2.40 & 6.84 & 2.78 & 4.86 & 4.50 & 4.77 \\
\hline D17 & 2.56 & 8.09 & 18.7 & 14.1 & 15.5 & 3.01 & 11.6 & 9.79 & 12.9 \\
\hline D20 & - & 3.48 & 11.4 & 8.08 & 11.8 & 4.60 & 8.14 & 7.21 & 8.33 \\
\hline D21 & - & - & - & - & 4.30 & 0.513 & 5.92 & 7.07 & 9.00 \\
\hline D22 & - & 4.64 & 10.8 & 6.88 & 10.3 & 3.93 & 7.18 & 6.39 & 7.66 \\
\hline D27 & - & 1.35 & 5.02 & 4.13 & 6.50 & 3.40 & 5.26 & 4.67 & 5.67 \\
\hline E45 & - & - & - & - & - & - & 0.958 & 1.83 & 2.95 \\
\hline E49 & - & 1.20 & 3.35 & 1.60 & 3.67 & 1.52 & 2.35 & 2.16 & 2.30 \\
\hline $\mathrm{F} 1$ & 44.6 & 48.6 & 94.8 & 120 & 66.6 & 27.8 & 62.2 & 60.8 & 71.4 \\
\hline F3 & 46.4 & 48.4 & 93.2 & 141 & 65.3 & 26.0 & 60.2 & 69.1 & 66.6 \\
\hline F6 & 40.3 & 41.7 & 116 & 91.0 & 59.3 & 23.1 & 60.7 & 44.1 & 65.0 \\
\hline \multirow[t]{2}{*}{ G6 } & - & - & 20.6 & 21.3 & 26.1 & 9.56 & 32.7 & 28.7 & 42.1 \\
\hline & $\mathrm{C}_{22}$ & $\mathrm{C}_{23}$ & $\mathrm{C}_{24}$ & $\mathrm{C}_{25}$ & $\mathrm{C}_{26}$ & $\mathrm{C}_{27}$ & $\mathrm{C}_{28}$ & $\mathrm{Sq}$ & $\mathrm{C}_{29}$ \\
\hline A10 & 2.67 & 4.68 & 3.10 & 4.62 & 3.00 & 6.82 & 6.97 & 76.0 & 14.6 \\
\hline A14 & 17.8 & 31.3 & 21.0 & 39.6 & 28.6 & 65.0 & 32.9 & 134 & 136 \\
\hline A24 & 7.91 & 13.5 & 9.42 & 15.0 & 10.2 & 22.6 & 12.4 & 161 & 48.4 \\
\hline A28 & 2.95 & 4.91 & 3.17 & 4.21 & 4.21 & 7.86 & 6.27 & 41.6 & 12.7 \\
\hline A31 & 13.1 & 21.2 & 13.1 & 24.7 & 15.4 & 37.2 & 24.1 & 93.4 & 80.4 \\
\hline A35 & 14.6 & 26.0 & 16.6 & 37.8 & 19.8 & 63.3 & 27.5 & 125 & 144 \\
\hline A37 & 16.2 & 28.6 & 18.8 & 36.8 & 22.6 & 56.1 & 33.7 & 197 & 117 \\
\hline A 40 & 9.38 & 14.3 & 9.67 & 17.4 & 13.8 & 30.7 & 22.2 & 83.2 & 60.2 \\
\hline A42 & 21.2 & 32.8 & 22.5 & 35.1 & 23.1 & 53.3 & 28.6 & 138 & 106 \\
\hline B7 & 7.54 & 10.5 & 7.42 & 9.01 & 6.39 & 10.2 & 7.95 & 92.5 & 17.1 \\
\hline B8 & 36.7 & 44.9 & 40.8 & 51.3 & 34.6 & 56.9 & 32.5 & 194 & 98.0 \\
\hline C6 & 47.4 & 89.6 & 58.3 & 144 & 66.4 & 237 & 104 & 100 & 565 \\
\hline $\mathrm{C} 7$ & 40.3 & 80.0 & 47.3 & 125 & 56.4 & 218 & 89.1 & 133 & 523 \\
\hline $\mathrm{C} 8$ & 21.4 & 35.9 & 27.0 & 42.0 & 22.9 & 55.6 & 30.7 & 51.8 & 127 \\
\hline $\mathrm{C} 10$ & 22.8 & 36.6 & 31.8 & 50.9 & 37.6 & 73.3 & 48.3 & 160 & 140 \\
\hline $\mathrm{C} 12$ & 45.3 & 89.0 & 69.1 & 156 & 90.2 & 262 & 135 & 141 & 587 \\
\hline $\mathrm{C} 14$ & 36.6 & 70.0 & 39.9 & 111 & 51.9 & 196 & 80.4 & 108 & 485 \\
\hline CA & 22.2 & 37.4 & 32.7 & 52.2 & 40.9 & 71.1 & 45.8 & 65.1 & 118 \\
\hline $\mathrm{CB}$ & 6.15 & 9.95 & 6.33 & 10.8 & 6.19 & 14.0 & 7.03 & 62.1 & 27.0 \\
\hline $\mathrm{CC}$ & 3.79 & 5.79 & 3.88 & 5.49 & 3.00 & 6.35 & 3.53 & 24.7 & 11.3 \\
\hline D7 & 54.0 & 90.7 & 58.2 & 123 & 63.8 & 213 & 105 & 119 & 512 \\
\hline D12 & 12.0 & 19.1 & 13.9 & 30.0 & 20.7 & 55.5 & 45.0 & 57.2 & 109 \\
\hline D16 & 4.80 & 8.32 & 5.12 & 8.00 & 3.94 & 10.8 & 5.54 & 50.3 & 22.2 \\
\hline D17 & 12.0 & 18.5 & 13.1 & 23.7 & 14.8 & 34.7 & 22.3 & 87.1 & 76.0 \\
\hline D20 & 7.66 & 12.4 & 9.93 & 16.4 & 8.57 & 21.0 & 12.5 & 70.1 & 43.1 \\
\hline D21 & 8.88 & 14.6 & 10.1 & 21.4 & 10.5 & 25.9 & 11.2 & 145 & 53.5 \\
\hline D22 & 6.69 & 10.8 & 7.10 & 12.3 & 6.39 & 14.0 & 12.8 & 78.4 & 25.8 \\
\hline D27 & 5.36 & 9.08 & 7.37 & 10.7 & 9.75 & 20.0 & 24.7 & 64.0 & 38.6 \\
\hline E45 & 3.42 & 5.86 & 3.93 & 6.36 & 4.00 & 9.80 & 4.61 & 12.5 & 17.9 \\
\hline E49 & 2.28 & 4.09 & 2.43 & 3.64 & 2.05 & 5.11 & 2.14 & 6.89 & 8.85 \\
\hline $\mathrm{F} 1$ & 70.5 & 85.6 & 57.9 & 83.9 & 58.9 & 116 & 73.4 & 111 & 201 \\
\hline F3 & 69.3 & 83.2 & 56.2 & 84.5 & 62.3 & 118 & 75.0 & 116 & 205 \\
\hline F6 & 65.4 & 95.8 & 67.0 & 131 & 74.9 & 205 & 98.3 & 275 & 457 \\
\hline \multirow[t]{2}{*}{ G6 } & 41.3 & 70.5 & 46.7 & 90.7 & 54.8 & 147 & 97.9 & 161 & 312 \\
\hline & $\mathrm{C}_{30}$ & $\mathrm{C}_{31}$ & $\mathrm{C}_{32}$ & $\mathrm{Di}$ & $\mathrm{C}_{33}$ & $\mathrm{C}_{34}$ & $\mathrm{C}_{35}$ & $\mathrm{UCM}$ & $\mathrm{CPI}$ \\
\hline A10 & 12.9 & 14.2 & 2.45 & 13.1 & 6.14 & 0.672 & 6.26 & 388 & 1.71 \\
\hline A14 & 60.1 & 167 & 21.4 & 57.7 & 79.6 & 9.46 & 47.8 & 2330 & 3.09 \\
\hline A24 & 42.9 & 51.0 & 8.64 & 28.8 & 19.9 & 2.28 & 24.5 & 1100 & 1.97 \\
\hline A28 & 12.6 & 11.1 & 3.84 & 6.92 & 4.98 & 1.12 & 6.25 & - & 1.41 \\
\hline A31 & 52.3 & 93.7 & 15.3 & 49.0 & 44.3 & 6.91 & 45.0 & 3410 & 2.40 \\
\hline A35 & 80.8 & 186 & 17.3 & 71.3 & 80.1 & 6.92 & 40.2 & 2180 & 3.26 \\
\hline A37 & 71.0 & 137 & 20.3 & 60.2 & 62.0 & 6.95 & 61.3 & 2980 & 2.56 \\
\hline A40 & 43.0 & 63.5 & 13.0 & 23.1 & 33.8 & 6.31 & 23.8 & 852 & 2.06 \\
\hline A42 & 41.6 & 126 & 19.0 & 42.3 & 60.3 & 9.26 & 44.2 & 3990 & 2.98 \\
\hline B7 & 11.2 & 17.4 & 4.68 & 10.7 & 9.59 & 1.78 & 10.9 & 658 & 1.83 \\
\hline $\mathrm{B} 8$ & 43.8 & 123 & 22.4 & 54.7 & 71.4 & 9.07 & 34.4 & - & 2.56 \\
\hline
\end{tabular}


Table 1 (continued)

\begin{tabular}{|c|c|c|c|c|c|c|c|c|c|}
\hline & $\mathrm{C}_{30}$ & $\mathrm{C}_{31}$ & $\mathrm{C}_{32}$ & $\mathrm{Di}$ & $\mathrm{C}_{33}$ & $\mathrm{C}_{34}$ & $\mathrm{C}_{35}$ & UCM & CPI \\
\hline C6 & 102 & 778 & 68.0 & 156 & 357 & 24.4 & 108 & 9250 & 5.46 \\
\hline C7 & 129 & 747 & 77.1 & 141 & 330 & 20.8 & 101 & 6400 & 5.05 \\
\hline $\mathrm{C} 8$ & 23.0 & 174 & 23.4 & 42.3 & 75.9 & 9.16 & 32.0 & 6560 & 4.05 \\
\hline $\mathrm{C} 10$ & 43.5 & 168 & 29.1 & 48.2 & 83.0 & 14.2 & 53.1 & 5490 & 2.85 \\
\hline $\mathrm{C} 12$ & 120 & 796 & 82.3 & 142 & 340 & 31.6 & 128 & 7920 & 4.49 \\
\hline $\mathrm{C} 14$ & 77.0 & 648 & 57.4 & 98.2 & 280 & 18.8 & 85.2 & 5120 & 5.82 \\
\hline CA & 43.1 & 138 & 28.3 & 39.9 & 69.2 & 13.8 & 49.5 & 2960 & 2.48 \\
\hline $\mathrm{CB}$ & 8.93 & 31.3 & 5.38 & 24.5 & 14.0 & 1.69 & 14.2 & 1300 & 3.10 \\
\hline $\mathrm{CC}$ & 4.05 & 13.9 & 2.05 & 6.19 & 6.33 & 1.19 & 5.98 & - & 2.89 \\
\hline D7 & 108 & 687 & 82.2 & 167 & 298 & 21.3 & 97.6 & 9420 & 4.61 \\
\hline D12 & 46.8 & 131 & 36.7 & 35.0 & 66.4 & 21.2 & 32.0 & 1300 & 2.35 \\
\hline D16 & 5.33 & 25.7 & 3.73 & 14.6 & 11.4 & 1.54 & 6.46 & - & 3.60 \\
\hline D17 & 27.0 & 90.9 & 18.9 & 42.6 & 44.9 & 8.30 & 29.3 & 1940 & 2.89 \\
\hline D20 & 20.7 & 49.7 & 9.11 & 33.1 & 22.4 & 4.36 & 13.7 & 1210 & 2.64 \\
\hline D21 & 20.4 & 62.0 & 12.6 & 41.1 & 29.6 & 7.94 & 18.3 & 1290 & 3.02 \\
\hline D22 & 6.88 & 29.9 & 4.56 & 26.0 & 12.0 & 0.972 & 11.0 & 911 & 2.73 \\
\hline D27 & 31.8 & 56.6 & 36.7 & 20.2 & 42.2 & 14.3 & 17.4 & 1250 & 1.48 \\
\hline E45 & 4.42 & 22.3 & 3.05 & 2.64 & 9.43 & 1.30 & 3.62 & - & 3.54 \\
\hline E49 & 2.12 & 11.0 & 1.18 & 2.30 & 4.60 & 0.455 & 2.55 & - & 3.77 \\
\hline $\mathrm{F} 1$ & 81.3 & 239 & 41.5 & 75.6 & 129 & 20.7 & 46.4 & 4580 & 2.63 \\
\hline F3 & 64.4 & 247 & 48.8 & 70.4 & 134 & 19.0 & 96.8 & 6660 & 2.75 \\
\hline F6 & 104 & 607 & 90.1 & 166 & 308 & 28.8 & 112 & 5820 & 4.12 \\
\hline G6 & 76.8 & 431 & 51.2 & 110 & 219 & 18.1 & 79.7 & 3530 & 3.84 \\
\hline
\end{tabular}

${ }^{\text {a }} \mathrm{Pr}$, pristane; $\mathrm{Ph}$, phytane; $\mathrm{Sq}$, squalene; Di, diploptene; $\mathrm{UCM}$, unresolved complex mixtures; $\mathrm{CPI}$, carbon preference index $=(1 / 2)\left[\left(n \mathrm{C}_{25}+n \mathrm{C}_{27}+\right.\right.$ $\left.\left.n \mathrm{C}_{29}+n \mathrm{C}_{31}+n \mathrm{C}_{33}\right) /\left(n \mathrm{C}_{24}+n \mathrm{C}_{26}+n \mathrm{C}_{28}+n \mathrm{C}_{30}+n \mathrm{C}_{32}\right)+\left(n \mathrm{C}_{25}+n \mathrm{C}_{27}+n \mathrm{C}_{29}+n \mathrm{C}_{31}+n \mathrm{C}_{33}\right) /\left(n \mathrm{C}_{26}+n \mathrm{C}_{28}+n \mathrm{C}_{30}+n \mathrm{C}_{32}+n \mathrm{C}_{34}\right)\right]$.

Table 2

Total organic carbon (TOC in $\left.\mathrm{gC}(100 \mathrm{~g})^{-1}\right)$ in East China Sea and southern Okinawa Trough sediments

\begin{tabular}{lllllll}
\hline \multicolumn{2}{l}{ East China Sea } & & & & \multicolumn{2}{l}{ Southern Okinawa Trough } \\
\cline { 1 - 1 } \cline { 6 - 6 } Sample & TOC & Sample & TOC & & Sample & TOC \\
\hline A10 & 0.059 & CA & 0.20 & OT1 & 0.65 \\
A14 & 0.34 & CB & 0.10 & OT3 & 0.56 \\
A24 & 0.18 & CC & 0.057 & OT4 & 0.79 \\
A28 & 0.046 & D7 & 0.49 & OT5 & 0.75 \\
A31 & 0.45 & D12 & 0.23 & OT8 & 0.71 \\
A35 & 0.21 & D16 & 0.082 & OT9 & 0.73 \\
A37 & 0.31 & D17 & 0.17 & OT12 & 0.81 \\
A40 & 0.15 & D20 & 0.098 & OT18 & 0.82 \\
A42 & 0.26 & D21 & 0.13 & & OT19 & 0.59 \\
B7 & 0.063 & D22 & 0.11 & & OT20 & 0.60 \\
B8 & 0.31 & D27 & 0.064 & & OT22 & 0.85 \\
C6 & 0.42 & E45 & 0.047 & OT23 & 0.76 \\
C7 & 0.42 & E49 & 0.043 & & \\
C8 & 0.16 & F1 & 0.46 & & \\
C10 & 0.24 & F3 & 0.43 & & \\
C12 & 0.45 & F6 & 0.52 & & \\
C14 & 0.33 & G6 & 0.46 & & \\
\hline
\end{tabular}

with Euclidean distances of 6.246, 7.034 and 8.717, respectively, indicating that the three components had very weak relation with other hydrocarbons. As a consequence, these three components were excluded for performing PCA. The score plot of the first two components explaining $74 \%$ of the total variance is shown in Fig. 3. To facilitate discussion, peripheral samples of all
OT samples were connected by line segments, forming a polygon; those in positive $\mathrm{PC} 1$ and negative $\mathrm{PC} 2$ as well as those in negative $\mathrm{PC} 1$ were, respectively, connected in the same way. Therefore, three groups are visible in the score plot, those virtually with a positive score on $\mathrm{PC} 1$ and with low positive and low negative scores on PC2 (group I), those with a positive score on $\mathrm{PC} 1$ and a high negative score on PC2 (group II), and those with negative scores on PC1 (group III). Of the three groups, group I contained 12 OT samples and three inner shelf samples (C8, D7 and F6). With the exception of sample D27, group II comprised seven samples on the inner shelf. The majority of samples (21) on the ECS shelf located away from the inner shelf constituted group III. In addition, samples F1 and F3, situated in high positive scores on PC1 and high positive scores on PC2, were fairly peculiar and might be considered outliers.

It is of importance to note that group I defined by 12 OT samples includes three samples on the inner shelf; this is especially unreasonable because group I clusters with distant samples instead of nearby samples. This requires more discussion. The Lanyang River (nearest river to the SOT, not shown) has been reported to be not a major hydrocarbon source for OT sediments, but to a less extent it does influence two stations OT 1 and OT 12 closest to the river mouth based on the individual TAR (terrigenous/ aquatic ratio) and CPI values (Jeng and Huh, 2006). This also reflects in OT1 in Fig. 3 that the sample is situated relatively away from the cluster of other OT samples. That is, sample OT1 has a low similarity to the other OT 
Table 3

Hydrocarbon concentrations $\left(\mathrm{ng} \mathrm{g}^{-1}\right)$ for Okinawa Trough (OT) sediments (from Jeng and Huh, 2006)

\begin{tabular}{|c|c|c|c|c|c|c|c|c|c|}
\hline \multirow[t]{2}{*}{ Sample } & \multicolumn{9}{|c|}{ Aliphatic hydrocarbons ${ }^{\mathrm{a}}$} \\
\hline & $\mathrm{C}_{15}$ & $\mathrm{C}_{16}$ & $\mathrm{C}_{17}$ & $\operatorname{Pr}$ & $\mathrm{C}_{18}$ & $\mathrm{Ph}$ & $\mathrm{C}_{19}$ & $\mathrm{C}_{20}$ & $\mathrm{C}_{21}$ \\
\hline OT1 & 1.94 & 29.3 & 97.6 & 170 & 110 & 45.9 & 116 & 85.1 & 87.5 \\
\hline OT3 & - & - & 23.8 & 28.1 & 61.4 & 23.2 & 55.7 & 50.3 & 49.2 \\
\hline OT4 & - & - & 42.7 & 49.4 & 81.1 & 26.2 & 96.1 & 75.4 & 82.4 \\
\hline OT5 & - & - & 20.1 & 17.9 & 56.7 & 17.9 & 66.6 & 70.7 & 82.1 \\
\hline OT8 & - & 32.7 & 90.4 & 118 & 88.7 & 28.7 & 95.1 & 71.5 & 77.8 \\
\hline OT9 & - & - & 18.3 & 17.1 & 55.7 & 15.8 & 89.5 & 83.1 & 95.9 \\
\hline OT12 & 80.3 & 63.8 & 103 & 147 & 89.1 & 32.0 & 102 & 65.5 & 64.7 \\
\hline OT18 & 10.7 & 42.9 & 103 & 101 & 103 & 36.3 & 79.5 & 73.8 & 73.1 \\
\hline OT19 & 11.2 & 32.2 & 67.2 & 89.8 & 65.5 & 20.5 & 52.2 & 47.7 & 56.5 \\
\hline OT20 & 10.8 & 28.2 & 52.7 & 34.1 & 52.0 & 15.7 & 40.2 & 28.6 & 36.0 \\
\hline OT22 & - & 14.5 & 80.6 & 113 & 101 & 37.9 & 104 & 78.8 & 84.5 \\
\hline \multirow[t]{2}{*}{ OT23 } & - & - & 14.9 & 15.6 & 49.4 & 17.4 & 54.6 & 56.5 & 60.7 \\
\hline & $\mathrm{C}_{22}$ & $\mathrm{C}_{23}$ & $\mathrm{C}_{24}$ & $\mathrm{C}_{25}$ & $\mathrm{C}_{26}$ & $\mathrm{C}_{27}$ & $\mathrm{C}_{28}$ & $\mathrm{Sq}$ & $\mathrm{C}_{29}$ \\
\hline OT1 & 96.4 & 137 & 114 & 171 & 111 & 241 & 165 & 217 & 418 \\
\hline OT3 & 53.2 & 70.6 & 65.0 & 98.6 & 54.8 & 136 & 87.6 & 178 & 253 \\
\hline OT4 & 88.7 & 125 & 98.6 & 156 & 96.7 & 243 & 168 & 199 & 473 \\
\hline OT5 & 86.7 & 122 & 97.3 & 149 & 95.4 & 220 & 157 & 271 & 382 \\
\hline OT8 & 82.3 & 119 & 96.8 & 150 & 96.3 & 221 & 145 & 161 & 421 \\
\hline OT9 & 108 & 135 & 116 & 156 & 97.6 & 214 & 139 & 317 & 392 \\
\hline OT12 & 65.1 & 103 & 97.6 & 160 & 102 & 240 & 176 & 184 & 457 \\
\hline OT18 & 72.3 & 107 & 77.7 & 148 & 79.7 & 214 & 149 & 230 & 451 \\
\hline OT19 & 69.8 & 94.7 & 73.5 & 110 & 73.0 & 168 & 88.3 & 100 & 319 \\
\hline OT20 & 41.2 & 99.0 & 77.3 & 178 & 94.8 & 346 & 121 & 99.0 & 690 \\
\hline OT22 & 86.6 & 125 & 99.6 & 145 & 101 & 236 & 141 & 28.2 & 433 \\
\hline \multirow[t]{2}{*}{ OT23 } & 64.4 & 94.7 & 78.6 & 133 & 76.4 & 195 & 127 & 171 & 359 \\
\hline & $\mathrm{C}_{30}$ & $\mathrm{C}_{31}$ & $\mathrm{C}_{32}$ & $\mathrm{Di}$ & $\mathrm{C}_{33}$ & $\mathrm{C}_{34}$ & $\mathrm{C}_{35}$ & $\mathrm{UCM}$ & CPI \\
\hline OT1 & 195 & 564 & 150 & 450 & 273 & 65.9 & 167 & 8930 & 2.35 \\
\hline OT3 & 102 & 378 & 77.9 & 874 & 167 & 25.9 & 121 & 3410 & 2.82 \\
\hline OT4 & 202 & 634 & 178 & 1130 & 295 & 76.3 & 186 & 8080 & 2.46 \\
\hline OT5 & 148 & 561 & 162 & 363 & 280 & 72.0 & 179 & 4450 & 2.46 \\
\hline OT8 & 168 & 573 & 153 & 917 & 263 & 69.8 & 182 & 8760 & 2.53 \\
\hline OT9 & 161 & 574 & 131 & 1310 & 260 & 55.8 & 165 & 8790 & 2.61 \\
\hline OT12 & 172 & 616 & 121 & 586 & 278 & 49.1 & 203 & 7420 & 2.72 \\
\hline OT18 & 106 & 606 & 127 & 626 & 265 & 44.4 & 206 & - & 3.23 \\
\hline OT19 & 97.9 & 456 & 80.0 & 915 & 206 & 26.4 & 94.9 & - & 3.25 \\
\hline OT20 & 114 & 885 & 91.1 & 79.9 & 371 & 26.4 & 101 & - & 5.24 \\
\hline OT22 & 195 & 618 & 167 & 716 & 316 & 76.9 & 206 & 10700 & 2.53 \\
\hline OT23 & 147 & 549 & 115 & 1210 & 230 & 34.8 & 177 & 3540 & 2.81 \\
\hline
\end{tabular}

${ }^{\mathrm{a}} \mathrm{Pr}$, pristine; $\mathrm{Ph}$, phytane; $\mathrm{Sq}$, squalene; $\mathrm{Di}$, diploptene; $\mathrm{UCM}$, unresolved complex mixtures; $\mathrm{CPI}$, carbon preference index $=(1 / 2)\left[\left(n \mathrm{C}_{25}+n \mathrm{C}_{27}+\right.\right.$ $\left.\left.n \mathrm{C}_{29}+n \mathrm{C}_{31}+n \mathrm{C}_{33}\right) /\left(n \mathrm{C}_{24}+n \mathrm{C}_{26}+n \mathrm{C}_{28}+n \mathrm{C}_{30}+n \mathrm{C}_{32}\right)+\left(n \mathrm{C}_{25}+n \mathrm{C}_{27}+n \mathrm{C}_{29}+n \mathrm{C}_{31}+n \mathrm{C}_{33}\right) /\left(n \mathrm{C}_{26}+n \mathrm{C}_{28}+n \mathrm{C}_{30}+n \mathrm{C}_{32}+n \mathrm{C}_{34}\right)\right]$

samples. If sample OT1 is excluded from Fig. 3, the area of group I is greatly reduced, and samples C8, D7 and F6 are not included in group I. In other words, the relation between these three samples and group I depends entirely on sample OT1, and it can be concluded that this relation is very weak, or even does not exist at all.

It is of great interest to examine the hydrocarbon distribution in the southwestern part of the ECS since it is an important area proposed for transporting POM from the ECS to the SOT by Liu et al. (2000). In their conceptual model, the possible route of transporting sediment is from the Changjiang River mouth, southward along the south- eastern coast of China, eastward across northern Taiwan Strait, along offshore northern Taiwan to the SOT. Considering the samples collected from the Changiiang River mouth to the SOT (22 samples on the shelf west of longitude $123^{\circ}$ and 12 in the SOT), it was found that only three samples (C8, D7 and F6) clustered with group I (OT samples) and the other 19 samples do not. This suggests that the hydrocarbon relation between the inner shelf and the SOT is actually weak, implying that the possibility of transporting hydrocarbons along the route of the conceptual model to SOT is very low. This result can be quite reliable because $n$-alkanes are relatively unreactive and 


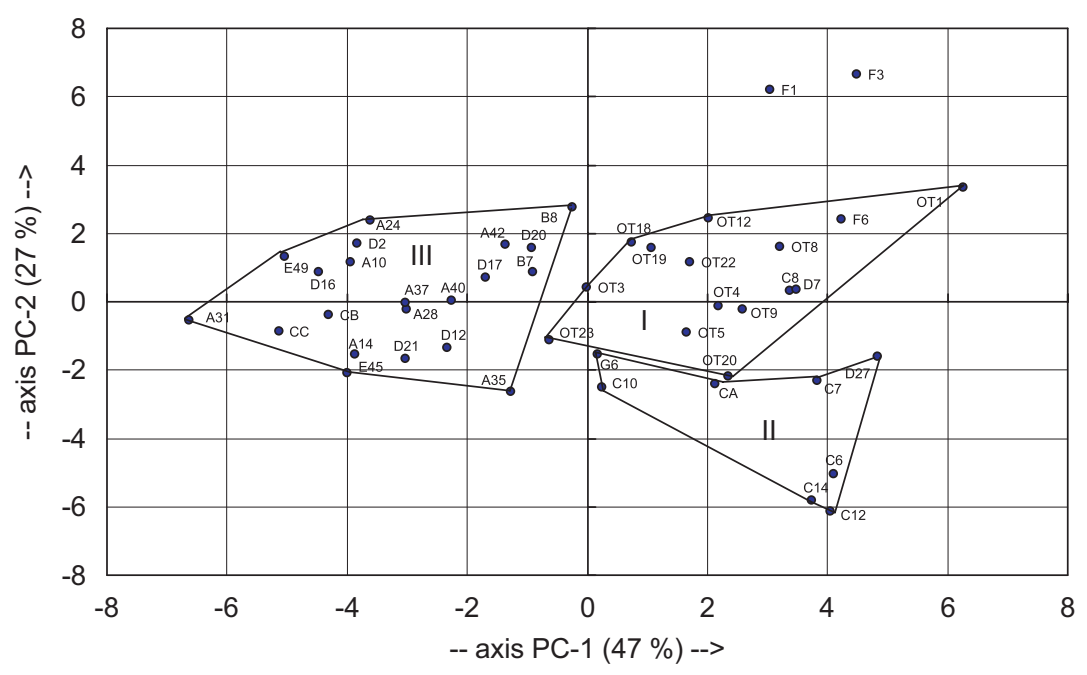

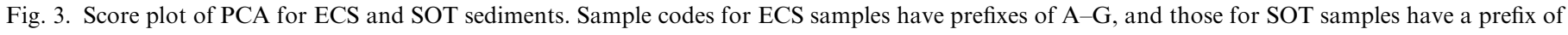

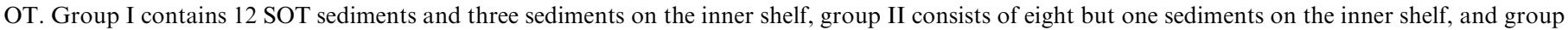
III is composed of 21 sediments mainly on the mid-to-outer shelf.

unlikely to be affected by any mechanism that would be selective for alternating homologs in the sequence (Hedges and Prahl, 1993).

One marked feature is that groups II and III are, respectively, located in positive and negative $\mathrm{PC}$, signifying their difference in hydrocarbon distribution. This is supported by the literature result that Changjiang sediments have been transported to the south via the coastal current along the Chinese coast and that offshore transport has been prevented by tidal currents and by the northward movement of the TWC (Milliman et al., 1989). Another reason may be that the inner shelf consists largely of muddy sediments, which are considered recent deposits, and the mid-to-outer shelf is composed chiefly of sandy sediments (Huh and $\mathrm{Su}, 1999)$, which are known as relict sediments (Niino and Emery, 1961). In addition, stations F1 and F3 located near the inner shelf have no relation with group II, which might be attributed to the influence of the TWC (Fig. 1). Furthermore, sample locations G6, F1 and F3 are very close to each other geographically (Fig. 1), but sample G6 separates clearly from samples F1 and F3 in the score plot (Fig. 3), suggesting that the deposition of Changjiang River sediments terminates at station G6.

As expected, groups I and III are separated from each other in Fig. 3, likely attributable to little material exchange between them. The KC flows northward and turns northeastward when approaching the ECS shelf although it intrudes onto the shelf in the winter and spring time (Chern and Wang, 1992; Hsueh et al., 1992).

Note that the SOT contains 12 stations within a relatively small area, but the hydrocarbon distribution of the samples varies widely. For example, the areas covered by groups I and III in Fig. 3 are comparable, but their respective geographical areas in the SOT and on the midto-outer shelf differ greatly (Fig. 1). The reason can be explained as follows. The lipid inputs by suspended matter/ sediment transport to the seas off northeastern Taiwan can derive from three sources (Fig. 1). From the northwest, a small alongshore flow just off northern Taiwan (south of station D27) along with the eddy flow (Tang et al., 1999) may carry materials from the southern ECS and Taiwan Strait to the SOT. The Lanyang River (from the west) exerts small influence on the SOT sediments as discussed earlier. Another source from south is other river runoffs from eastern Taiwan carried by the Kuroshio Current to the SOT.

Terrestrial higher plant wax contribution to sediment or soil is generally expressed by the CPI of $n$-alkanes. Higher $\mathrm{CPI}_{25-33}$ values indicate greater contribution from vascular plants (Rieley et al., 1991); $\mathrm{CPI}_{25-33}$ values close to one are thought to indicate greater input from marine microorganisms, recycled organic matter, and/or petroleum (Kennicutt et al., 1987). The present $\mathrm{CPI}_{25-33}$ values of the ECS ranged widely from 1.41 to 5.82 with an average of 3.12 (calculated from Table 1), suggesting non-uniform distribution of terrigenous hydrocarbons. In general, high CPI values were found on the inner shelf, and low values on the mid-toouter shelf. This suggests that the Changjiang River contributes more to the former than to the latter, consistent with the literature result indicated earlier. On the other hand, the average CPI value $(3.12, n=34)$ of the ECS was comparable to that (average $2.92, n=12$ ) of the SOT. These two averages are considered to be relatively high, suggesting that fossil fuel contamination is minimal. However, it should be noted that CPI values would become smaller if there is any addition of 'old' hydrocarbons (e.g., CPI of petroleum close to 1; Bray and Evans, 1961) to the sediments from pollution. Some samples were found to contain different proportions of 'old' hydrocarbons as evidenced by the presence of $17(\alpha) \mathrm{H}, 21(\beta) \mathrm{H}$ hopanes. This fossil component could be contributed from fossil fuels through contamination (Albaigés and Albrecht, 
1979; Shaw et al., 1985; Readman et al., 1996) from boat traffic or a spill. The addition of 'old' hydrocarbons will lower some CPI values observed in ECS sediments. The purpose of this study is to compare hydrocarbon distribution between ECS and SOT and to explore if they are related. For comparing the two areas, eight samples (C14, C12, C10, C8, C7, C6, D7 and F6; Fig. 1) on the ECS inner shelf and 12 samples in the SOT were chosen; their average CPIs were, respectively, $4.56 \pm 0.93$ and $2.92 \pm 0.79$, suggesting that the hydrocarbon sources to the two areas were quite different. The result is quite dependable because the CPI is a diagenetically insensitive ratio (Hedges and Prahl, 1993), implying that the CPI would not change over time during transport.

The ratio of terrigenous-to-aquatic $n$-alkanes were estimated as

$\mathrm{TAR}=\left(n C_{27}+n C_{29}+n C_{31}\right) /\left(n C_{15}+n C_{17}+n C_{19}\right)$.

This ratio is valuable for determining changes in relative contributions of organic matter from land and aquatic flora although it may over-represent the absolute amounts from terrigenous sources (Meyers, 1997). This ratio was adopted in the present study to estimate the relative contribution of terrigenous and marine sources to ECS and SOT sediments. The average TAR for the ECS inner shelf was $50.4 \pm 58.0 \quad(n=8)$, and that for the SOT was $9.64 \pm 4.33(n=12)$ (calculated from Tables 1 to 3 ). This suggests that the two areas receive different hydrocarbon inputs. The TAR values are expected to be larger for SOT sediments than for ECS sediments (since the SOT is much deeper than the ECS and since aquatic components degrade faster) if they have the same source. The present result is reversed and suggests the ECS and SOT having different sources. Note that this ratio is one of the factors used for comparing the differences of the multiple study sites. Higher plant $n$-alkanes were the predominant components in the sediments from the ECS and SOT; to correct for the grain size effect, $\left(n \mathrm{C}_{27}+n \mathrm{C}_{29}+n \mathrm{C}_{31}\right) /$ TOC was used to estimate the input of higher plant $n$-alkanes to the two areas (Prahl, 1985). The SOT and ECS inner shelf gave $\left(n \mathrm{C}_{27}+n \mathrm{C}_{29}+n \mathrm{C}_{31}\right) /$ TOC averages of $1731 \pm 482 \times$ $10^{-7} \mathrm{~g}(\mathrm{~g} \mathrm{C})^{-1}(n=12)$ and $3016 \pm 868 \times 10^{-7} \mathrm{~g}(\mathrm{~g} \mathrm{C})^{-1}$ $(n=8)$ (calculated from Tables 1 to 3 ), respectively, indicating different inputs of higher plant $n$-alkanes to the two areas. In the HMW region, the $\mathrm{C}_{27}$ and $\mathrm{C}_{29}$ $n$-alkanes are diagnostic of waxes from trees and shrubs, and the $n-\mathrm{C}_{31}$ alkane is diagnostic of grass waxes (Cranwell, 1973). The $n \mathrm{C}_{31} /\left(n \mathrm{C}_{27}+n \mathrm{C}_{29}+n \mathrm{C}_{31}\right)$ ratio was employed to see if any difference existed between the two areas. The average ratios of $n \mathrm{C}_{31} /\left(n \mathrm{C}_{27}+n \mathrm{C}_{29}+n \mathrm{C}_{31}\right)$ for the SOT and ECS inner shelf were, respectively, $0.478 \pm 0.012(n=12)$ and $0.483 \pm 0.018(n=8)$ (calculated from Tables 1 to 3 ), showing no difference between the two averages. This can be attributed to $n$-alkane distribution maximizing at $\mathrm{C}_{31}$, which makes the change in $\mathrm{C}_{31}$ insensitive.
Seven stations (not shown) between Taiwan and 12 SOT sample sites were selected for collecting total suspended matter ( $2 \mathrm{~m}$ below sea surface) on July 23-26, 2000 (cruise \#590). The average CPI of the seven samples was $1.01 \pm 0.12$. This low CPI average reflects the contribution from a nearby river (Lanyang River), which is known to contain "old" hydrocarbons in its suspended matter and sediments (Jeng and Kao, 2002; Jeng and Huh, 2006). In addition, one of our cruises (\#525 on August 17-24, 1998) to the ECS happened to be the high runoff period of the Changjiang River. The runoff of the river had a very different color than the offshore seawater of the ECS. Two stations $-\mathrm{C} 14$ and $\mathrm{C} 15\left(31^{\circ} 19.96^{\prime} \mathrm{N}, 122^{\circ} 30.09^{\prime} \mathrm{E}\right.$, not shown) representing the runoff of the river were occupied, and one sample of total suspended matter $(2 \mathrm{~m}$ below sea surface) for each station was collected. CPI values of the two stations were 1.55 and 1.32 , which differed greatly from those of off northeast Taiwan. These results indicate that transport of suspended sediment from the Changjiang River mouth to the SOT is quite unlikely.

In conclusion, most sediments on the ECS inner shelf did not group with those in the SOT, indicating dissimilarity in hydrocarbon distribution between the two areas. Moreover, striking differences in CPI, TAR and $\left(n \mathrm{C}_{27}+n \mathrm{C}_{29}+n \mathrm{C}_{31}\right) /$ TOC values were found between SOT and ECS inner shelf. In addition, hydrocarbon distribution differences in suspended matter from Changjiang River runoff and off northeastern Taiwan were also observed, suggesting that hydrocarbon sources to the two areas were different. Based on these results, it can be concluded that hydrocarbon contribution from the ECS inner shelf to the SOT appears to be minimal.

\section{Acknowledgments}

Our special thanks go to the captain, crew and technicians of the R/V Ocean Researcher I for help with sediment collection. We are grateful to Prof. Philip A Meyers (University of Michigan) and one anonymous reviewer for constructive comments and suggestions. This study was supported by a National Science Council Grant (NSC88-2611-M-002-017-K2) of the Republic of China.

\section{References}

Albaigés, J., Albrecht, P., 1979. Fingerprinting marine pollutant hydrocarbons by computerized gas chromatography-mass spectrometry. International Journal of Environmental Analytical Chemistry 6, 171-190.

Beardsley, R.C., Limeburner, R., Yu, H., Cannon, G.A., 1985. Discharge of the Changjiang (Yangtze River) into the East China Sea. Continental Shelf Research 4, 57-76.

Bieger, T., Abrajano, T.A., Hellou, J., 1997. Generation of biogenic hydrocarbons during a spring bloom in Newfoundland coastal (NW Atlantic) waters. Organic Geochemistry 26, 207-218.

Biscaye, P.E., Anderson, R.F., Deck, B.L., 1988. Fluxes of particles and constituents to the eastern United States continental slope and rise: SEEP-I. Continental Shelf Research 8, 855-904. 
Blumer, M., Guillard, R.R.L., Chase, T., 1971. Hydrocarbons of marine phytoplankton. Marine Biology 8, 183-189.

Bouloubassi, I., Saliot, A., 1993. Investigation of anthropogenic and natural organic inputs in estuarine sediments using hydrocarbon markers (NAH, LAB, PAH). Oceanologica Acta 16, 145-161.

Bray, E.E., Evans, E.D., 1961. Distribution of $n$-paraffins as a clue to recognition of source beds. Geochimica et Cosmochimica Acta 22, $2-15$.

Chen, M.P., Lin, K.L., Huang, C.K., 1995. Sedimentary structure and texture of surficial sediment off northeastern Taiwan. Ti-Chih (ROC) 15, 15-47 (in Chinese with English abstract).

Chern, C.S., Wang, J., 1992. On the season variation of the Kuroshio intrusion onto the East China Sea. Acta Oceanographica Taiwanica 29, 1-17.

Chung, Y., Chang, W.C., 1995. Pb-210 fluxes and sedimentation rates on the lower continental slope between Taiwan and the South Okinawa Trough. Continental Shelf Research 15, 149-164.

Cranwell, P.A., 1973. Chain-length distribution of $n$-alkanes from lake sediments in relation to postglacial environment change. Freshwater Biology 3, 259-265.

DeMaster, D.J., McKee, B.A., Nittrouer, C.A., Qian, J.C., Cheng, G.D., 1985. Rates of sediment accumulation and particle reworking based on radiochemical measurements from continental shelf deposits in the East China Sea. Continental Shelf Research 4, 143-158.

Gagosian, R.B., Peltzer, E.T., 1986. The importance of atmospheric input of terrestrial organic material to deep sea sediments. Organic Geochemistry 10, 661-669.

Gaudette, H.E., Flight, W.R., Toner, L., Folger, D.W., 1974. An inexpensive titration method for the determination of organic carbon in recent sediments. Journal of Sedimentary Petrology 44, 249-253.

Gough, M.A., Rowland, S.J., 1990. Characterization of unresolved complex mixtures of hydrocarbons in petroleum. Nature 344, 648-650.

Hedges, J.I., Prahl, F.G., 1993. Early diagenesis: consequences for applications of molecular biomarkers. In: Engel, M.H., Macko, S.A. (Eds.), Organic Geochemistry: Principles and Applications. Plenum Press, New York, pp. 237-253.

Hsueh, Y., Wang, J., Chern, C.S., 1992. The intrusion of the Kuroshio across the continental shelf northeast of Taiwan. Journal of Geophysical Research 97 (C9), 14323-14330.

Huh, C.A., Su, C.C., 1999. Sedimentation dynamics in the East China Sea elucidated from ${ }^{210} \mathrm{~Pb},{ }^{137} \mathrm{Cs}$ and ${ }^{239,240} \mathrm{Pu}$. Marine Geology 160, 183-196.

Jeng, W.L., Chen, M.P., 1995. Grain size effect on bound lipids in sediments off northeastern Taiwan. Organic Geochemistry 23, 301-310.

Jeng, W.L., Huh, C.A., 2004. Lipids in suspended matter and sediments from the East China Sea Shelf. Organic Geochemistry 35, 647-660.

Jeng, W.L., Huh, C.A., 2006. A comparison of sedimentary aliphatic hydrocarbon distribution between the southern Okinawa Trough and a nearby river with high sediment discharge. Estuarine, Coastal and Shelf Science 66, 217-224.

Jeng, W.L., Kao, S.J., 2002. Lipids in suspended matter from the humandisturbed Lanyang River, northeastern Taiwan. Environmental Geology 43, 138-144.

Kao, S.J., Lin, F.J., Liu, K.K., 2003. Organic carbon and nitrogen contents and their isotopic compositions in surficial sediments from the East China Sea shelf and the southern Okinawa Trough. Deep-Sea Research II 50, 1203-1217.

Katayama, H., Watanabe, Y., 2003. The Huanghe and Changjiang contribution to seasonal variability in terrigenous particulate load to the Okinawa Trough. Deep-Sea Research II 50, 475-485.

Kennicutt II, M.C., Barker, C., Brooks, J.M., DeFreitas, D.A., Zhu, G.H., 1987. Selected organic matter source indicators in the Orinoco, Nile and Changjiang deltas. Organic Geochemistry 11, 41-51.

Liu, K.K., Iseki, K., Chao, S.Y., 2000. Continental margin carbon fluxes. In: Hanson, R.B. (Ed.), The Changing Ocean Carbon Cycle, IGBP Book Series. Cambridge University Press, Cambridge, pp. 187-239.

Liu, K.K., Peng, T.H., Shaw, P.T., Shiah, F.K., 2003. Circulation and biogeochemical processes in the East China Sea and the vicinity of
Taiwan: an overview and a brief synthesis. Deep-Sea Research II 50, $1055-1064$

Matsumoto, G., 1983. Comparative study on organic constituents in polluted and unpolluted inland aquatic environments-V. Organic carbons and hydrocarbons in sediments. Water Research 17, 823-830.

Meyers, P.A., 1997. Organic geochemical proxies of paleoceanographic, paleolimnologic, and paleoclimatic processes. Organic Geochemistry 27, 213-250.

Meyers, P.A., Leenheer, M.J., Eadie, B.J., Maule, S.J., 1984. Organic geochemistry of suspended and settling particulate matter in Lake Michigan. Geochimica et Cosmochimica Acta 48, 443-452.

Milliman, J.D., Meade, R.H., 1983. World-wide delivery of river sediment to the oceans. The Journal of Geology 91, 1-21.

Milliman, J.D., Shen, H.T., Yang, Z.S., Meade, R.H., 1985. Transport and deposition of river sediment in the Changjiang estuary and adjacent continental shelf. Continental Shelf Research 4, 37-45.

Milliman, J.D., Qin, Y.S., Park, Y.A., 1989. Sediments and sedimentary processes in the Yellow and East China Seas. In: Taira, A., Masuda, F. (Eds.), Sedimentary Facies in the Active Plate Margin. Terra Scientific Publishing Company, Tokyo, pp. 233-249.

Niino, H., Emery, K.O., 1961. Sediments of shallow portions of East China Sea and South China Sea. Geological Society of America Bulletin 72, 731-762.

ODP, 2001. Seafloor observatories and the Kuroshio Current. Ocean Drilling Program, Leg 195 Preliminary Report. Available at: 〈http:// www-odp.tamu.edu/publications/prelim/195_prel/195toc.html $\rangle$.

Oguri, K., Matsumoto, E., Yamada, M., Saito, Y., Iseki, K., 2003. Sediment accumulation rates and budgets of depositing particles of the East China Sea. Deep-Sea Research II 50, 513-528.

Prahl, F.G., 1985. Chemical evidence of differential particle dispersal in the southern Washington coastal environment. Geochimica et Cosmochimica Acta 49, 2533-2539.

Prahl, F.G., Hayes, J.M., Xie, T.M., 1992. Diploptene: an indicator of terrigenous organic carbon in Washington coastal sediments. Limnology and Oceanography 37, 1290-1300.

Readman, J.W., Mantoura, R.F.C., Rhead, M.M., 1987. A record of polycyclic aromatic hydrocarbons (PAH) pollution obtained from accreting sediments of the Tamar Estuary, UK: evidence for nonequilibrium behavior of PAH. The Science of the Total Environment 66, 73-94.

Readman, J.W., Bartocci, J., Tolosa, I., Fowler, S.W., Oregioni, B., Abdulraheem, M.Y., 1996. Recovery of the coastal marine environment in the Gulf following the 1991 war-related oil spills. Marine Pollution Bulletin 32, 493-498.

Rieley, G., Collier, R.J., Jones, D.M., Eglinton, G., 1991. The biogeochemistry of Ellesmere Lake, UK-I: Source correlation of leaf wax inputs to the sedimentary lipid record. Organic Geochemistry 17, 901-912.

Rohmer, M., Bouvier-Nave, P., Ourisson, G., 1984. Distribution of hopanoid triterpenes in prokaryotes. Journal of General Microbiology $130,1137-1150$.

Salas, N., Ortiz, L., Gilcoto, M., Varela, M., Bayona, J.M., Groom, S., Álvarez-Salgado, X.A., Albaigés, J., 2006. Fingerprinting petroleum hydrocarbons in plankton and surface sediments during the spring and early summer blooms in the Galician coast (NW Spain) after the Prestige oil spill. Marine Environmental Research 62, 388-413.

Shaw, D.G., Hogan, T.E., McIntosh, D.J., 1985. Hydrocarbons in the sediments of Port Valdez, Alaska: consequences of five years' permitted discharge. Estuarine, Coastal and Shelf Science 21, 131-144.

Tang, T.Y., Hsueh, Y., Yang, Y.J., Ma, J.C., 1999. Continental slope flow northeast of Taiwan. Journal of Physical Oceanography 29, 1353-1362.

Tolosa, I., Bayona, J.M., Albaigés, J., 1996. Aliphatic and polycyclic aromatic hydrocarbons and sulfur/oxygen derivatives in northwestern Mediterranean sediments: spatial and temporal variability, fluxes and budgets. Environmental Science and Technology 30, 2495-2503.

Venkatesan, M.I., Brenner, S., Ruth, E., Bonilla, J., Kaplan, I.R., 1980. Hydrocarbons in age-dated sediment cores from two basins in the 
Southern California Bight. Geochimica et Cosmochimica Acta 44, 789-802.

Walsh, J.J., Biscaye, P.E., Csanady, G.T., 1988. The 1983-1984 Shelf Edge Exchange Processes (SEEP)-I. Experiment: hypotheses and highlights. Continental Shelf Research 8, 435-456.

Winters, K., Parker, P.L., Van Baalen, C., 1969. Hydrocarbons of bluegreen algae: geochemical significance. Science 158, 467-468.
Wong, G.T.F., Chao, S.Y., Li, Y.H., Shiah, F.K., 2000. The Kuroshio Edge Exchange Processes (KEEP) study - an introduction to hypotheses and highlights. Continental Shelf Research 20, 335-347.

Yanagi, T., Takahashi, S., Hoshika, A., Tanimoto, T., 1996. Seasonal variation in the transport of suspended matter in the East China Sea. Journal of Oceanography 52, 539-552. 